\title{
A Chopped Neural Front-end featuring Input Impedance Boosting with suppressed offset-induced charge transfer
}

\author{
Stefan Reich, Graduate Student Member, IEEE, Markus Sporer, Graduate Student Member, IEEE, \\ Maurits Ortmanns, Senior Member, IEEE \\ Institute of Microelectronics, University of Ulm, Ulm, Germany
}

\begin{abstract}
Modern neuromodulation systems typically provide a large number of recording and stimulation channels, which reduces the available power and area budget per channel. To maintain the necessary input-referred noise performance despite growingly rigorous area constraints, chopped neural front-ends are often the modality of choice, as chopperstabilization allows to simultaneously improve (1/f) noise and area consumption. The resulting issue of a drastically reduced input impedance has been addressed in prior art by impedance boosters based on voltage buffers at the input. These buffers precharge the large input capacitors, reduce the charge drawn from the electrodes and effectively boost the input impedance. Offset on these buffers directly translates into charge-transfer to the electrodes, which can accelerate electrode aging. To tackle this issue, a voltage buffer with ultra-low time-averaged offset is proposed, which cancels offset by periodic reconfiguration, thereby minimizing unintended charge transfer. This article explains the background and circuit design in detail and presents measurement results of a prototype implemented in a $180 \mathrm{~nm}$ HV CMOS process. The measurements confirm that signal-independent, buffer offset induced charge transfer occurs and can be mitigated by the presented buffer reconfiguration without adversely affecting the operation of the input impedance booster. The presented neural recorder front-end achieves state of the art performance with an area consumption of $0.036 \mathrm{~mm}^{2}$, an input referred noise of $1.32 \mu \mathrm{V}_{\text {rms }}(1 \mathrm{~Hz}$ to $200 \mathrm{~Hz})$ and $3.36 \mu \mathrm{V}_{\mathrm{rms}}(0.2 \mathrm{kHz}$ to $10 \mathrm{kHz})$, power consumption of $13.7 \mu \mathrm{W}$ from $1.8 \mathrm{~V}$ supply, as well as CMRR and PSRR $\geq 83 \mathrm{~dB}$ at $50 \mathrm{~Hz}$.
\end{abstract}

Index Terms-ASIC, Brain-Machine-Interfaces, BMI, Neural Recording, Bio-sensing, Biomedical Implant, Brain Implant, Impedance Boosting, Low-offset Buffer

\section{INTRODUCTION}

$\mathbf{I}$ NTEGRATED circuits for implantable electronics have greatly advanced over the last decade. Modern biomedical implants feature large numbers of bidirectional channels, combining both low noise recording and flexible, biphasic stimulation [1], [2]. Adapting stimulation parameters according to bio-markers extracted from the recorded neural data stream results in closed-loop neuromodulation, which is extensively researched for deployment in potential treatment

Paper received Feb 26, 2021; revised April 16, 2021.

This work was funded by the German Federal Ministry of Education and Research under research grant MR:Implant. We thank our industrial partner CorTec GmbH, 79108 Freiburg, Germany, for the electrode testing array.

All authors are with the Institute of Microelectronics at the University of Ulm, 89081 Ulm, Germany. (e-mail: stefan.reich@uni-ulm.de) options for neurological diseases like Parkinsons Disease and Epilepsy [3]-[6]. Further applications for brain-machineinterfaces (BMI) include brain research [7] and the development of brain-controlled prosthetics [8], [9].

The growing demand for larger channel counts imposes rigorous power and area constraints on the individual channel and requires minimized electrode arrays. Area benefits due to technology scaling are not always exploitable in neuromodulation SoCs, as the implemented stimulator requires highvoltage (HV) compliant devices for efficient charge delivery into the tissue [10], [11], which are not readily available in smaller technology nodes. The bio-signals of interest are usually divided into two categories: Local field potentials (LFPs, $0.2 \mathrm{~Hz}$ to $200 \mathrm{~Hz}$ ) and action potentials (APs/Spikes, $200 \mathrm{~Hz}$ to $5000 \mathrm{~Hz}$ ). The input-referred noise performance is widely regarded a key metric for neural recording frontends, and is adversely affected by both the aggravating area and power budgets. A decrease in available power results in additional thermal noise contribution, while reductions in the total area increase the 1/f noise. To overcome these limitations, chopper-stabilized neural front-ends are popular in recent state-of-the-art (SotA) designs [3], [12]-[15].

The chopper however comes with the disadvantage of a significantly reduced input impedance [16], resulting in increased charge transfer into the electrodes. This can accelerate electrode aging, and attenuates the input signal thereby degrading the SNR, which counteracts the initial effort for improved noise performance. Numerous publications have addressed the issue and proposed solutions to improve the input impedance in chopped neural front-ends. In [12] and [17], a positive feedback loop was used to boost the input impedance; [14] reported issues of feedbackbased impedance boosters in presence of a DSL, due to the cancellation of low-frequency content at the output node. Still, the DSL is crucial for suppression of electrode dc offsets (EDO) towards the output. Therefore, an auxiliary boosting path with voltage buffers was introduced in [14], and refined in [15]. This input impedance boosting based on the auxiliary paths effectively minimizes the charge drawn from the electrodes by precharging the large input capacitors for a short duration instantly after every edge of the chopping clock. Disadvantageously, buffer offset results in unintended charge-transfer to the electrodes, thus minimizing the buffer 
offset is crucial. To address this issue, a voltage buffer with ultra-low, time-averaged offset was proposed in [18] and is elaborated in this work including prototype implementation and measurement results.

The article is organized as follows: Section 2 reviews the analysis on the auxiliary path input impedance boosting scheme, and derives equations and models. In Section 3, the self-compensated voltage buffer is presented, alongside simulation results and implementation considerations. Section 4 presents the implementation of a chopped neural front-end featuring the proposed architecture of the auxiliary path input impedance boosting. Sections 5 and 6 present measurement results and a state-of-the-art comparison, respectively. Section 7 concludes the paper.

\section{Analysis of the Auxiliary Path InPut IMPEDANCE BOOSTING SCHEME}

\section{A. Chopped neural front-ends and input impedance}

When chopper-stabilization is utilized to improve the $1 / \mathrm{f}$ noise performance of neural recording front-ends, the first chopper is typically placed in front of the input capacitors. This architecture can operate without large off-chip capacitors [19], which is crucial in implantable systems where printed circuit board (PCB) area is very limited. Figure 1 shows an implementation of a conventional (black) chopperstabilized bio-signal recorder front-end.

Chopping the low noise amplifier (LNA) provides the first amplification without superimposing $1 / \mathrm{f}$ noise in the band of interest. The gain is set by the capacitor ratio $A=\frac{C_{\mathrm{in}}}{C_{\mathrm{fb}}}$ and is typically in the range of $20 \mathrm{~dB}$ to $40 \mathrm{~dB}$, constituting a tradeoff between noise performance and harmonic distortion. A dc servo loop (DSL) is implemented to add a very low-frequency (sub-hertz) high-pass corner (e.g. [15]) by feeding back the low-frequency content of the signal, effectively canceling it at the input node. This is required as electrode offset could otherwise saturate the recorder stage. The range of allowable electrode offsets can be controlled by the ratio $\frac{C_{\mathrm{dsl}}}{C_{\text {in }}}$. Extended cancellation range is bought at the cost of increased noise contribution of the DSL. Typically, the electrode dc offset is in the range of $\pm 100 \mathrm{mV}$, and is predominantly caused by mismatches in the electrode-tissue interface, (geometrical) differences between individual electrodes and varying rates of electrode aging and degradation.

Following every edge of the chopping clock, the large input capacitors $C_{\text {in }}$ must be recharged by the connected electrodes, resulting in a drastic reduction in input impedance [18]:

$$
R_{\text {in }}^{*}=\frac{1}{2 \cdot C_{\text {in }} \cdot f_{\text {chop }}}
$$

Eq. (1) describes a switched-capacitor equivalent resistance. A large input impedance is though required to firstly avoid SNR degradation due to the parasitic voltage divider formed by electrode and the front-end circuitry, and to secondly minimize electrode offset induced dc currents which

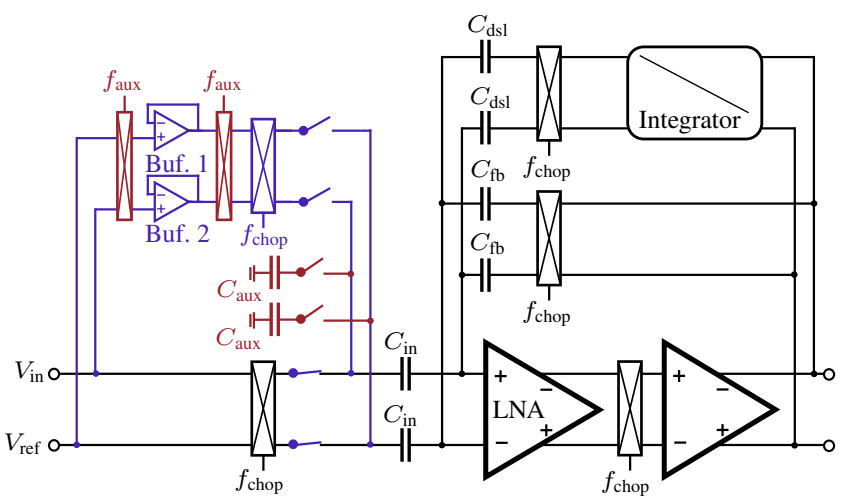

Fig. 1: State of the art chopped neural recorder, without input impedance boosting in black, with auxiliary path [14] in blue and improved aux. path input impedance boosting [15] in red

could result in both tissue and electrode damage. Input impedances reported in recent publications are in the range of several tens or hundreds of $\mathrm{M} \Omega$ and above [12], [20], [21].

\section{B. Analysis of auxiliary path input impedance boosting}

Figure 1 (blue) also depicts the auxiliary path input impedance boosting scheme from [14]. The direct input path between the electrodes and the input capacitors is disconnected for a small fraction of every chopping cycle. During this time, the large input capacitors are precharged through the auxilliary path buffers, which drastically reduces the charge drawn from the electrodes after the precharging phase and thus boosts the effective input impedance. Due to the exponential charging characteristic, a majority of the total charge in $C_{\text {in }}$ is transfered within a short timeframe immediately after the clock edge, thus a significant increase in input impedance can be achieved even with a short precharge phase; measured results for this mechanism report a $15 x$ boost in input impedance in [14].

Any difference between the electrode voltage and the buffer output at the end of the precharging phase decreases the booster effectiveness as charge is now drawn from the electrode to load $C_{\text {in }}$ to the intended voltage. Therefore, the most relevant buffer non-idealities are finite gain-bandwidth and offset, and their influence is analyzed in the following.

1) Effect of Buffer Offset: Given the limited input range, the buffer offset can be assumed to be constant and signalindependent, with a deterministic (by design) and a random (mismatch-induced) component. The operation of the auxiliary path input impedance booster can be split into 4 repetitive phases, which are depicted in Figure 2, where the excess charge induced by buffer offset is highlighted in red. Phase a) shows the precharging of the capacitors, starting on a chopping clock edge. The offset, which is w.l.o.g. only modeled in buffer $B 2$, results in excess charge on $C_{\mathrm{in}, 1}$, which is given by $Q_{\mathrm{ex}}=V_{\text {offset }} \cdot C_{\mathrm{in}}$. This charge is transfered to the electrode connected to $V_{\text {in }}$ once the precharging has 


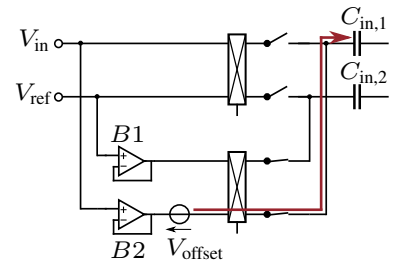

a)

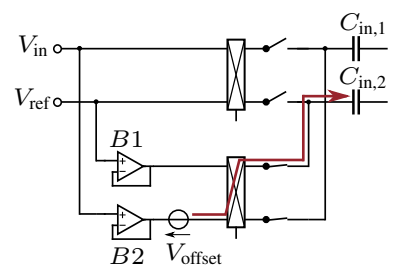

c)

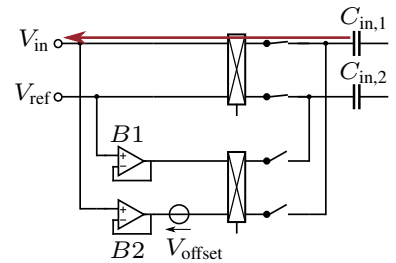

b)

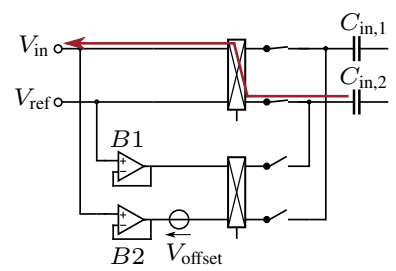

d)
Fig. 2: The 4 phases of aux. path input impedance boosting: Precharging in a) and c), electrode connected in b) and d). Offset-induced excess charge transfer is highlighted in red.

concluded, as is depicted in Fig. 2 b). The same procedure of excess charge accumulation and transfer to the electrode is observed for the other clock phase, which is illustrated in $c$ ) and $d$ ). Please note that in this implementation the excess charge introduced by the single-ended offset of buffer $B 2$ is always transfered to the same electrode $V_{\text {in }}$, regardless of the chopper phase, and thus on average results in a dc current into the electrode. As it is independent from the input signal, it can be modeled as constant current source [18]

$$
I_{\mathrm{dc}-\mathrm{offset}}=Q_{\mathrm{ex}} \cdot 2 \cdot f_{\mathrm{chop}}=\frac{V_{\mathrm{offset}}}{R_{\mathrm{in}}^{*}}
$$

where $R_{\text {in }}^{*}$ is the non-boosted, chopper dominated input impedance in Eq. (1).

2) Effect of finite Gain-Bandwidth: Finite gain-bandwidth (GBW) of the auxiliary path buffers leads to incomplete settling, which is aggravated when very short precharging phases and large input capacitors are used. At the end of a precharging phase of the auxiliary path, the resulting signaldependent error is compensated by the electrode, which leads again to charge transfer. The time-averaged current is proportional to the input signal, and can thus be modeled as equivalent resistance. Let the voltage over the input capacitor after the precharging be given by $V_{\text {buf }}$, then the relative settling error is $x=1-\frac{V_{\text {buf }}}{V_{\text {in }}}$. This $x$ depends on several system parameters, namely the transconductance of the buffer, the capacitance of $C_{\text {in }}$ and the duration $t_{\text {pre }}$ of the precharging phase, resulting in $x=\exp \left(-\frac{t_{\mathrm{pre}} \cdot g_{m}}{C_{\text {in }}}\right)$ for a single-pole buffer amplifier; the relative settling error $x$ is thus used as an intuitive abbreviation in the following. This yields the effective signal-dependent current and the equivalent input impedance [18] as

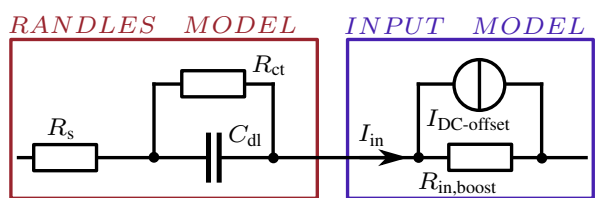

Fig. 3: Simple electrode and input impedance model

$$
\begin{aligned}
I_{\mathrm{R}_{\text {in,boost }}} & =V_{\text {in }} \cdot x \cdot 2 \cdot C_{\text {in }} \cdot f_{\text {chop }} \\
R_{\text {in,boost }} & =\frac{1}{x \cdot 2 \cdot C_{\text {in }} \cdot f_{\text {chop }}}=\frac{1}{x} \cdot R_{\text {in }}^{*}
\end{aligned}
$$

with $R_{\text {in }}^{*}$ from Eq. (1).

It is important to keep in mind that the voltage $V_{\text {in }}$ and in extension the current $I_{\mathrm{R}_{\mathrm{in}, \text { boost }}}$ are dominated by the large electrode dc offset and not by the comparatively tiny neural signal amplitude.

Note that an offset and bandwidth limitation of buffer B1 has the same effect on the second electrode $V_{\text {ref }}$.

\section{Input Impedance Model}

This analysis of the non-ideal auxiliary path input impedance booster yields a simple model of the resulting boosted input impedance, consisting of an equivalent resistance, limited by the GBW-induced relative settling error $x$ of the auxilliary path boosters, and a parallel current source modelling the signal-independent charge transfer due to buffer offsets. The total input current $I_{\text {in }}$ is then the sum of the currents through both parallel devices, thus $I_{\text {in }}=I_{\text {dc-offset }}+I_{\mathrm{R}_{\text {in,boost }}}$. The model is depicted in Fig. 3 [18].

\section{Improved auxiliary path input impedance booster}

In order to counteract these non-idealities, the auxiliary path input impedance booster was improved in [15], which is shown in Fig. 1 (red). The additional choppers around the buffer stage are clocked with a slower clock, which is an integer fraction $\frac{1}{n}$ of $f_{\text {chop. As a result, the auxiliary buffers }}$ switch position every $n$ cycles, and the buffer offset induced current $I_{\mathrm{dc}-\text { offset }}$ is determined by the average offset of both buffers. Unfortunately, deterministic offsets in both buffers will not be reduced at all, and the standard deviation of stochastic offsets will be statistically reduced only by $\sqrt{2}$ through averaging.

The additional capacitors $C_{\text {aux }}$ in Fig. 1 are used in [15] to aid the voltage settling by providing an additional charge reservoir for a very fast initial settling. This is bought at a large area penalty, with $C_{\text {aux }}=8 \cdot C_{\text {in }}$, but is successful in providing an additional boost of 2.66 -fold, thereby improving the total impedance boost from $29 x$ to $76 x$ [15]. 


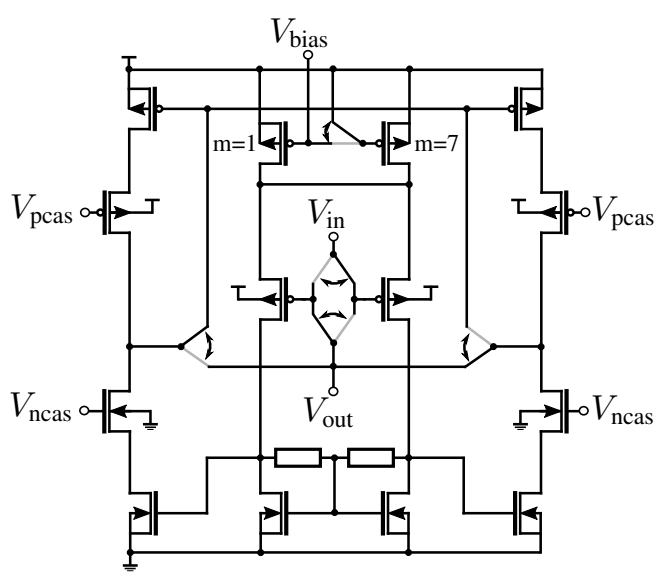

Fig. 4: Proposed Buffer Amplifier Architecture

\section{Self-COMPEnSATEd Voltage Buffer}

The method from [15], described in Sec. II-D, significantly improves the buffer settling and thereby increases $R_{\text {in,boost }}$ as compared to the groups prior work in [14], but the buffer offset is only slightly improved resulting in minor reductions of $I_{\mathrm{dc}-\text { offset }}$. Therefore, the proposed implementation focuses on minimizing buffer offset, and addresses the issue by utilizing a buffer architecture for ultra-low time-averaged offset [18].

When chopping the two auxiliary path buffers in Fig. 1 (red part), the resulting averaged buffer offset is still a random variable, and thus the effectiveness of the averaging is unpredictable: If both buffers happen to have identical offsets, then permuting their position produces no benefit at all. If, however, the buffers have offsets of equal magnitude but opposite sign, then averaging results in optimal cancellation. Eventually, any constellation between these borderline cases is possible, and as random mismatch-induced offsets are unknown prior to fabrication, the effectiveness of the offset reduction can vary.

A deterministic and reliable way of compensating buffer offset is therefore favorable. Inferring from the above considerations, optimal cancellation is achieved by averaging any random offset with its own negative. This can be achieved by periodic reconfiguration of a single buffer: Periodically inverting the offset results in its ideal cancellation, regardless of the random offset's magnitude. Following this idea, a buffer featuring self-compensation through periodic reconfiguration, resulting in ultra-low time-averaged offset, was introduced in [18] and is depicted in Figure 4.

The architecture is based on a symmetrical operational amplifier in order to minimize deterministic offsets. In a single-ended configuration, one of the two outer branches has a diode-connection and the other one is the output. Switching the diode connection allows to redefine this assignment, while simultaneously changing the signs of the amplifier inputs. During a reconfiguration, the diode branch becomes
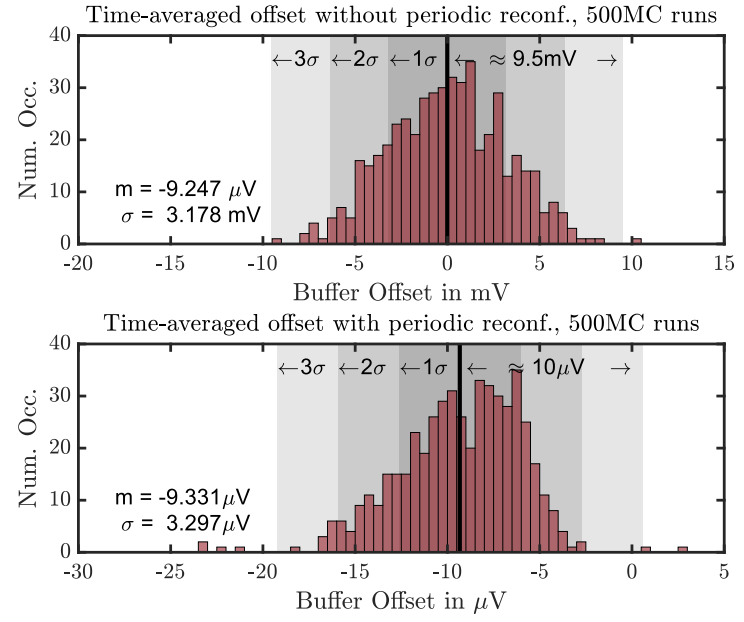

Fig. 5: MC Analysis of self-compensated buffer offset, with and without reconfiguration

the output and vice versa. The new output is fed back to what is now the negative input to preserve the unity-gain operation. The input voltage $V_{\text {in }}$ is then connected to the new positive input, which completes the reconfiguration. In this way, the whole architecture is virtually mirrored, except for random mismatch based differences of the individual devices. While normal buffer operation resumes unaltered, the random offset is inverted and can thus be mitigated by time-averaging. The reconfiguration can take place during a low-power idle phase, as the application requires active operation with large gain-bandwidth only during the short precharging phase. This way, significant reductions in total power consumption are possible.

Figure 5 shows a histogram of buffer offsets extracted from 500 Monte Carlo simulation runs of the circuit in Fig. 4, which was implemented in a $180 \mathrm{~nm}$ HV-CMOS technology with a $1.8 \mathrm{~V}$ supply. Plotted in the top plot is the proposed buffer architecture with disabled reconfiguration, while the bottom plot depicts the same setup with periodic reconfiguration enabled. The $3 \sigma$ range of $9.5 \mathrm{mV}$ for the reconfiguration-less buffer setup (top half) could be improved by $\sqrt{2}$ via averaging of two buffers (see Sec. II-D [15]), resulting in a minor improvement of $3 \sigma=6.7 \mathrm{mV}$. However, by employing the self-compensation method for periodic offset inversion detailed above, the $3 \sigma$ range of time-averaged buffer offsets can be reduced by approximately three orders of magnitude, from $9.5 \mathrm{mV}$ to $9.9 \mu \mathrm{V}$ (bottom half). Superposing any mismatch-induced random offset, also a deterministic offset e.g. from finite common mode rejection ration (CMRR) has to be expected. As the same amplifier is used in both simulations and the resulting deterministic offset is not affected by the self-compensation (and neither by averaging), it is visible as the same mean in both simulation results. The expected total offset, consisting of deterministic and random part, and with it the corresponding current 


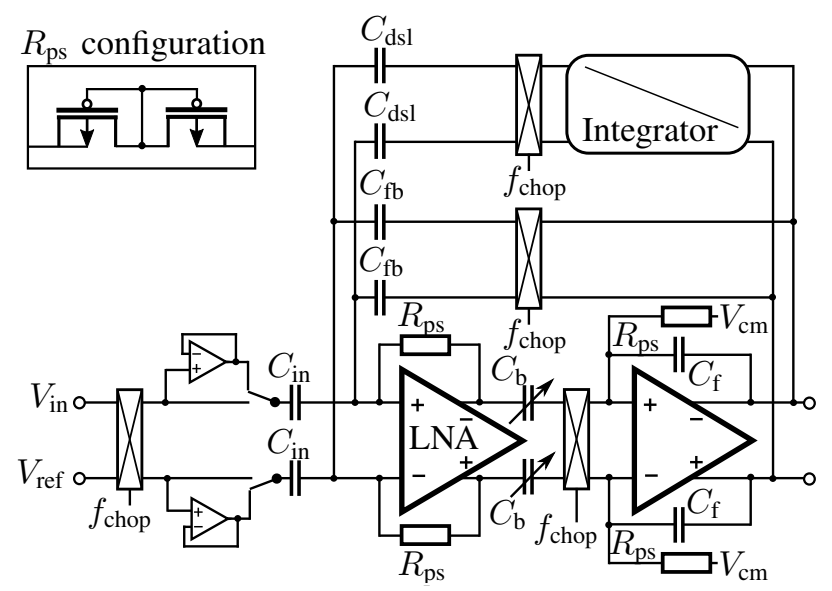

Fig. 6: Implemented neural recording front-end, featuring the presented buffer in a novel input impedance booster

$I_{\text {dc-offset, }}$, is thus reduced approximately $500 x$, from $<10 \mathrm{mV}$ to $<20 \mu \mathrm{V}$.

\section{NEURAL RECORDER IMPLEMENTATION}

The self-compensated buffer was incorporated in the design of a neural front-end in a $180 \mathrm{~nm}$ HV CMOS technology.

The overall architecture is depicted in Fig. 6 and was inspired by [15]. The implementation features an enhanced auxiliary path input impedance booster, which is simpler compared to [15] as it does not require mingling chopper control signals and precharging switch functionality. Instead, a separate clock signal for the buffer reconfiguration is used. Also, placing the buffers in the up-modulated signal domain prevents their $1 / \mathrm{f}$ noise from superposing the signal in the band of interest. The down-side is a slight decrease in input impedance, since the tiny parasitic input capacitor of the buffers $C_{\mathrm{p}}$ is behind the chopper, thus adding a rather large resistance $R_{\mathrm{p}}=\frac{1}{2 \cdot f_{\text {chop }} \cdot C_{\mathrm{p}}}$ in parallel to $R_{\text {in,boost. The self- }}$ compensated buffer does not rely on low intrinsic offset, but instead cancels mismatch-based offset dynamically. Therefore, the input devices do not require large area for matching and their parasitic gate-capacitance can be negligibly small (simulated: $C_{\mathrm{p}} \approx 15.4 \mathrm{fF}$ ), especially when compared to the large input capacitor of $C_{\mathrm{in}}=4 \mathrm{pF}$. The total input impedance is then given by $R_{\text {in,total }}=R_{\text {in,boost }} \| R_{\mathrm{p}}$. With $f_{\text {chop }}=20 \mathrm{kHz}$ and a realistic assumption of the settling error, e.g. $x \approx 1$ to $3 \%, R_{\text {in,boost }} \approx 200$ to $600 \mathrm{M} \Omega$ is expected according to Eq. (4). This is significantly smaller than the limitation due to the chopped buffer $\left(R_{\mathrm{p}}=1.62 \mathrm{G} \Omega\right)$ and therefore $R_{\mathrm{in} \text {,total }} \approx R_{\mathrm{in} \text {,boost. Thus, placing the chopper }}$ in front of the buffers has negligible adverse effect on the input impedance for the given system parameters.

Using an approach similar to that illustrated in Fig. 2 provides an intuitive insight into the functionality of the architecture by tracing offset-induced excess charge from the buffers to the electrodes. Each buffer is always associated with a specific input capacitor, but the connected electrode changes on each chopping cycle. Thus, the total charge per time transfered to the electrode is affected by both buffers. Each of the buffers uses the presented self-compensation to periodically invert its own offset, such that it cancels on the electrode on time-average, preventing charge accumulation. Therefore, the effective $I_{\mathrm{dc}-\text { offset }}$ is proportional to the averaged residual buffer offset, which is minimal due to the reconfiguration.

The further components in the front-end architecture shown in Fig. 6 include an LNA, which is implemented as an inverter-based amplifier. This allows for very favorable $\frac{g m}{I_{\mathrm{D}}}$, as both the NMOS and the PMOS contribute to the overall $g m$, thereby improving thermal noise performance while respecting the tight power restrictions. The disadvantage of worse $1 / \mathrm{f}$ noise performance due to the NMOS input device is mitigated through the chopper-stabilization. The $C_{\mathrm{b}}$ capacitors are used to reduce chopping ripples [22], as LNA offset is prevented from up-modulation in the following chopper. In contrast to prior work [15], these capacitors are tunable in the presented implementation which allows for a programmable low-pass corner defined by $f_{\mathrm{LPC}}=\frac{C_{\mathrm{b}}}{C_{\mathrm{f}}} \cdot f_{\text {chop }}$.

The implementation is optimized for noise, thus a larger gain of $40 \mathrm{~dB}$ was used as compared to $26 \mathrm{~dB}$ [15]. As matching of the capacitors is crucial in this architecture to avoid CMRR degradation [23], the smallest capacitors $C_{\mathrm{fb}}$ in the design were chosen to be $40 \mathrm{fF}$, which improves robustness to mismatch, but also requires a large $C_{\text {in }}=$ $4 \mathrm{pF}$ for the targeted signal amplification, thereby adversely affecting $R_{\text {in }}^{*}$ from Eq. (1). This highlights a trade-off between CMRR, which benefits from improved matching [23] in non-minimum sized capacitors, and the input impedance $R_{\text {in,boost }}$, which depends more than proportionally on the input capacitor, as both $R_{\mathrm{in}}^{*}$ and the settling error $x$ in Eq. (4) improve for decreasing $C_{\mathrm{in}}$.

The dc operating points of the amplifiers are defined by pseudo-resistor paths to the input node; pseudo-resistors are also used for the DSL integrator. The problems with distortion due to the non-linearity of the pseudo-resistors were relaxed as a target spurious free dynamic range of $\geq$ $65 \mathrm{~dB}$ for a $5 \mathrm{mV}_{\mathrm{pp}}$ input signal is acceptable. The precharge assist capacitors $C_{\text {aux }}=8 \cdot C_{\text {in }}$ from prior work [15] were not used to keep the design area small. Instead, proper buffer settling and in extension sufficient $R_{\text {in,boost }}$ is achieved through longer precharging time and increased buffer GBW. The auxiliary path buffers each consume $1.1 \mu \mathrm{W}$ in idle mode and $4 \mu \mathrm{W}$ in active mode. With the $4 \mathrm{pF}$ input capacitor loading the buffer in active mode, a $-3 \mathrm{~dB}$ corner at $70 \mathrm{kHz}$ was simulated for the buffer.

\section{Measurement Results}

The circuit was prototyped in a $180 \mathrm{~nm}$ HV CMOS technology, as it is intended to be incorporated in an existing stimulator for a neuromodulator. A chip photo is shown in Fig. 7. The prototype allows to deactivate the impedance 


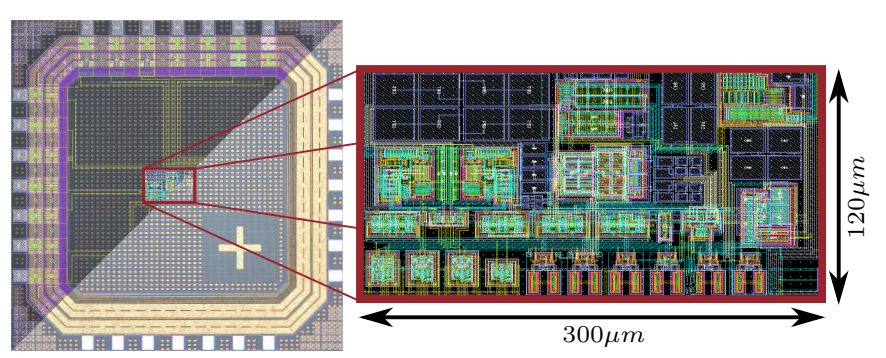

Fig. 7: Chip photograph with partial layout overlay and detailed neurorecorder layout

booster and the offset compensation in the auxiliary path buffers and also allows to vary the precharging time for evaluation purposes. The chopping frequency $f_{\text {chop }}$ was set to $20 \mathrm{kHz}$ for all presented measurements, which is a compromise between input impedance $\left(R_{\text {in,boost }} \propto \frac{1}{f_{\text {chop }}}\right)$ and the desired suppression of residual out-of-band chopping ripples (e.g. prior to sampling/digitization) with low-order filters which requires sufficient spectral distance from the signal band edge.

\section{A. Input Impedance Booster}

The auxiliary path boosted input impedance is measured over various voltages, as the model is resistive with current source. The input impedance $R_{\text {in }}$ is then given by the slope of the best fit line through the measured points according to $R_{\text {in }}=\left(\frac{\Delta I}{\Delta U}\right)^{-1}$.

Fig. 8 shows the measured input current over the input voltage with (red) and without (blue) input impedance boosting for a single device under test. For the unboosted case, the input impedance $R_{\text {in }}^{*} \approx 5.9 \mathrm{M} \Omega$, which is in fair accordance to the $6.25 \mathrm{M} \Omega$ that were expected nominally according to Eg. (1). The deviation of $\approx 6 \%$ is attributed to absolute capacitance process variation. Measurements of 3 additional samples revealed fairly constant results, with $R_{\text {in }}^{*} \in$ $[5.82,5.92] \mathrm{M} \Omega$. Activating the impedance booster results in a significantly reduced slope $R_{\text {in,boost }} \approx 175 \mathrm{M} \Omega$, which is a 30x increase over the unboosted dc input impedance for the plotted device. The measurements of the boosted input impedance for all 4 samples resulted in $R_{\text {in,boost }} \in[175,183]$ $\mathrm{M} \Omega$.

Considering only $R_{\text {in,boost }}$, which corresponds to the slope of the regression line, will however result in a faulty estimation of the total input current $I_{\text {in }}$ (see Fig. 3), as the buffer offset induced constant current component $I_{\mathrm{dc} \text {-offset }}$ in the derived model is ignored. While this additional constant current is irrelevant for the signal attenuation issue, $I_{\mathrm{dc} \text {-offset }}$ can be a major factor for the rate of electrode decay and must therefore be carefully considered. The effect of the offset induced constant current is significant as soon as $I_{\mathrm{dc} \text {-offset }} \approx I_{\mathrm{R}_{\mathrm{in}}}$, which is generally the case if $V_{\text {offset }} \approx V_{\mathrm{EDO}} \cdot x$ according to Eqs. (2) and (4). Assuming typical values, e.g. an electrode offset of up to $\pm 100 \mathrm{mV}$ and a settling error of

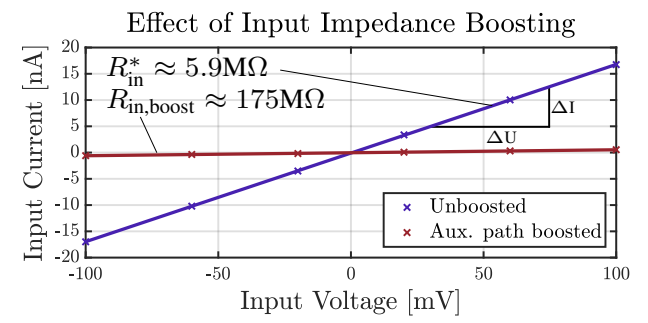

Fig. 8: Input current over voltage, showing the effect of the aux. path input impedance booster

$x=3 \%$, reveals that the offset induced constant current can be the dominant contributor of unintended charge transfer into the electrode in cases where the buffer offset exceeds $\pm 3 \mathrm{mV}$.

Fig. 9 shows the effect of the proposed buffer reconfiguration on the dc input current $I_{\text {in }}$. With periodic reconfiguration disabled, a significant input current is measured even for zero input voltage for this device under test, corresponding to the constant current $I_{\mathrm{dc} \text {-offset }} \approx 0.5 \mathrm{nA}$ for this device. This is depicted in the blue trace. The magnitude of $I_{\text {dc-offset }}$ is proportional to the buffer offset, which is dominated by the mismatch-induced random offset component when the buffer self-compensation is disabled (see Fig. 5). Thus, the constant current component was different for all 4 measured devices, with $I_{\text {dc-offset }} \in[0.1,0.5] \mathrm{nA}$. Eq. (2) allows to estimate the non-compensated aux. path buffer offset $V_{\text {offset }}=2.6 \mathrm{mV}$ of the plotted instance, which is within the $1 \sigma$ range according to the MC simulation (see Fig. 5). Activating the selfcompensation of the buffer mitigates its time-averaged offset, and accordingly the constant current $I_{\mathrm{dc}-\text { offset }}$ is minimized as well, which can be seen in the red trace. This was reliably achieved for all 4 measured samples. The buffer reconfiguration occurs every $n$ cycles of $f_{\text {chop, similar to }}$ the slower clock $f_{\text {aux }}$ in Sec. II-D [15]. This integer fraction $\frac{1}{n}$ is programmable in the presented prototype, and $n=2$ was selected for this measurement. As the buffer settling is unaltered, the resistive part of the input circuit model remains unchanged. But the offset induced dc current is reduced to a non-measurably small value. Accordingly, the slope and thus $R_{\text {in,boost }}$ are identical. These measurements prove both the derived model and the effectiveness of the self-compensation. Reproducing the results with multiple samples highlights the reliability.

Without offset compensation, an interesting constellation occurs at $V_{\text {in }} \approx-80 \mathrm{mV}$ for this particular device, as $V_{\text {in }}=R_{\text {in,boost }} \cdot I_{\text {dc-offset }}$, resulting in near-zero input current despite a considerable input voltage (see Fig. 9). Conducting measurements in this sweet spot would thus yield an almost infinite input impedance, which is however unrealistic as electrode dc offset is unknown and changes over time.

In order to see the effect of buffer settling accuracy on the boosted input impedance, the prototype allows to define a reconfigurable precharging phase. According to Eq. (4), the 


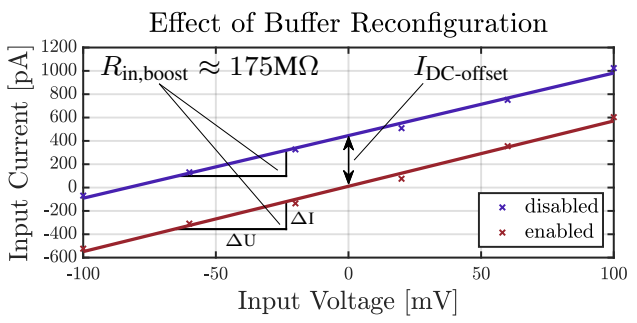

Fig. 9: Input current over voltage, showing the effect of buffer reconfiguration

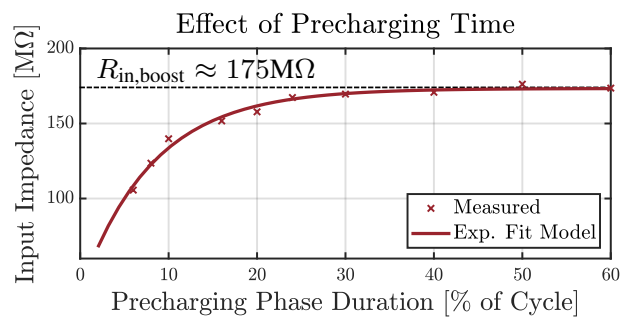

Fig. 10: Boosted input impedance over the relative duration of the precharge time

input impedance is proportional to the relative settling error $x$, which is itself a function of the $g m$ of the buffer, the input capacitor $C_{\text {in }}$ and the precharging duration.

Fig. 10 shows the measured input impedance over the duration of the precharging phase in percent of the cycle time. The shape of the exponential best fit curve meets the expectations according to Eq. (4): Large improvements of the input impedance $R_{\text {in,boost }}$ are gained for any increment of the precharging time at first, but the benefit soon decreases once the settling improves. The voltage settling on the capacitor follows an exponential characteristic, and so does the settling error dependent $R_{\text {in,boost }}$. Therefore the precharging phase can be limited in this implementation to around $30 \%$, as afterwards the settling does not yield considerable improvements. Keeping this phase as short as possible is beneficial for power consumption, as the buffers are idling; in [15] the buffer settling was limited to only $3.1 \%$, however with a much smaller $C_{\text {in }}$ and additional support by the huge $C_{\text {aux }}$, which led to higher area consumption in a much smaller CMOS technology. Thus, the optimum precharging strongly depends on the chosen parameters and implementation goals like $C_{\text {in }}$, power and area.

According to Fig. 10, saturation of the input impedance occurs at $175 \mathrm{M} \Omega$, which is significantly smaller than the 200 to $600 \mathrm{M} \Omega$ range derived in Sec. IV. The limitation is due to an additional effect which is independent of the buffers: Due to dc servo loop settling, the potential at the capacitive input node of the LNA, to which $C_{\text {in }}$ is connected, is still unsteady when the precharging phase concludes. Therefore, charge is drawn from the electrodes to $C_{\mathrm{in}}$ after the precharging phase, even if ideal precharge was possible. This effect scales with $C_{\text {in }}$ and $f_{\text {chop }}$, and thus results in a limit of the boosting factor,

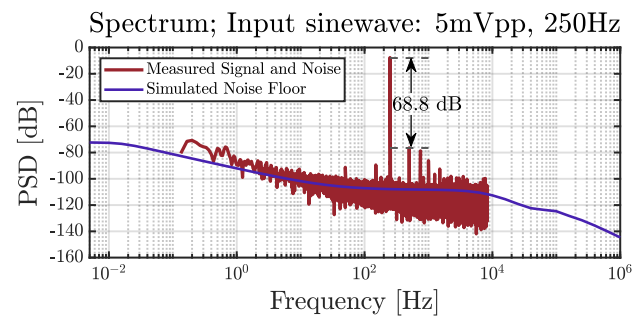

Fig. 11: Output spectrum of the neural front-end, displaying a spurious free range of $68.8 \mathrm{~dB}$, and simulated noise floor

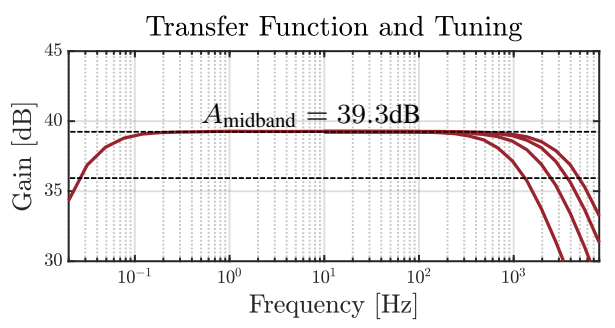

Fig. 12: Recorder transfer function, showing the DSL corner and the adaptable high-frequency cut-off

and not in a limit of the absolute input impedance $R_{\text {in,boost }}$. In the given implementation, the boosting factor is limited to $30 x$, which was consistently achieved for all measured samples.

\section{B. Neural Recorder}

Figure 11 shows the spectrum of the chopped neural recorder in presence of a $5 \mathrm{mVpp}$ sinusoidal input with $f=250 \mathrm{~Hz}$. The measured spurious free dynamic range is $68.8 \mathrm{~dB}$. Besides harmonic bins and a small $50 \mathrm{~Hz}$ peak, the influence of residual 1/f noise is clearly visible for smaller frequencies, while thermal noise is the dominant noise source for frequencies exceeding the $1 / \mathrm{f}$ noise corner at approximately $f_{\mathrm{nc}}=45 \mathrm{~Hz}$. The input-referred noise was measured at $1.32 \mu \mathrm{V}_{\text {rms }}$ (LFPs, $1 \mathrm{~Hz}$ to $200 \mathrm{~Hz}$ ) and $3.36 \mu \mathrm{V}_{\text {rms }}$ (APs, $200 \mathrm{~Hz}$ to $10000 \mathrm{~Hz}$ ), which slightly exceeds the simulated noise levels (blue trace, Fig. 11).

The adaptable low-pass filtering is shown in Figure 12. The $3 \mathrm{~dB}$ cut-off corners are at $1.25,2.5,3.75$ and $5 \mathrm{kHz}$, and scale with the chopping frequency, which allows adaptive anti-aliasing filtering (AAF). The definition of the cut-off frequency through a capacitor ratio provides very decent precision, but parasitic capacitances must be taken into account through analog-extracted design verification. Fig. 12 also shows the high-pass corner introduced by the DSL at $f_{\mathrm{hp}}=$ $25 \mathrm{mHz}$, which is required to allow low-frequency recording while suppressing electrode dc offset to prevent amplifier saturation. The DSL integrator is implemented using pseudoresistors, therefore measuring the high-pass corner allows to determine the resistance $R_{\mathrm{ps}}=50 \mathrm{~T} \Omega$. The implemented DSL manages to suppress electrode offsets of up to $\pm 100 \mathrm{mV}$ before linearity is adversely affected. 


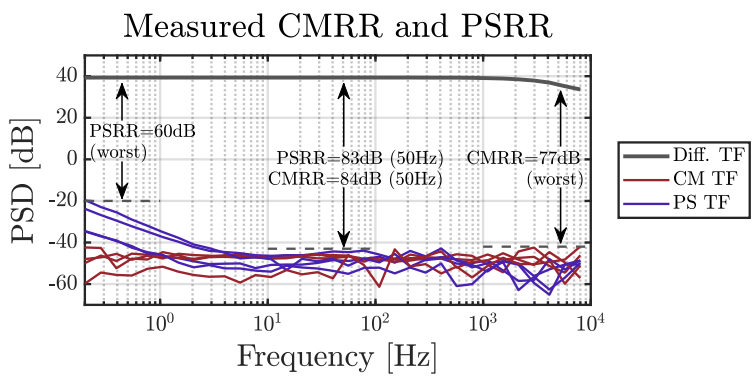

Fig. 13: Measured CMRR and PSRR of the neural recorder front-end (4 samples)

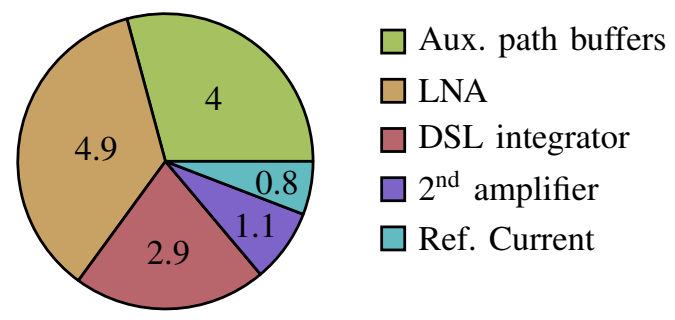

Fig. 14: Power consumption of individual components in $\mu \mathrm{W}$; total power $P=13.7 \mu \mathrm{W}$

Figure 13 shows the measured common mode rejection ratio and the power supply rejection ratio (PSRR). As both ratios depend strongly on random mismatch, data from 4 available samples is given. The PSRR degrades at frequencies lower than approximately $5 \mathrm{~Hz}$, resulting in a worst-case PSRR of $60 \mathrm{~dB}(0.2 \mathrm{~Hz})$. At the upper band-edge $(5 \mathrm{kHz})$, CMRR decreases due to the roll-off of the signal gain. Still, a worst case CMRR of $77 \mathrm{~dB}$ is measured. Both quantities are better than these worst-case value throughout most of the signal band, with PSRR $=83 \mathrm{~dB}$ and $\mathrm{CMRR}=84 \mathrm{~dB}$ at $50 \mathrm{~Hz}$.

The pie chart in Fig. 14 details how the total power $P=$ $13.7 \mu \mathrm{W}$ is shared by the individual components. The lowpower idle phases of the aux. path buffers allow to decrease the average power consumption, as a $30 \%$ active precharge phase is sufficient. Thus, with $30 \% \cdot P_{\text {buf,act }}+70 \% \cdot P_{\text {buf,idle }}=$ $30 \% \cdot 4 \mu \mathrm{W}+70 \% \cdot 1.1 \mu \mathrm{W}=1.97 \mu \mathrm{W}$, the average buffer power consumption is cut in half by the low-power mode. The other main power consumers are the LNA and the DSL integrator, both for thermal noise reasons.

\section{Verification by Electrode Measurement}

In order to validate the benchtop measurements, an electrode experiment was conducted and the results are depicted in Fig. 15. For this experiment, a 16-channel PtIr-electrode array of 4 diameters $(0.5,1.0,1.7$ and $2.7 \mathrm{~mm})$ was used (Courtesy of our industrial partners CorTec GmbH, Freiburg, Germany). Similar electrodes are typically used in LFP recordings, therefore the input signal was chosen as a $20 \mathrm{~Hz}$, $1 \mathrm{mV}_{\mathrm{pp}}$ sinewave. The electrodes were placed in phosphate-

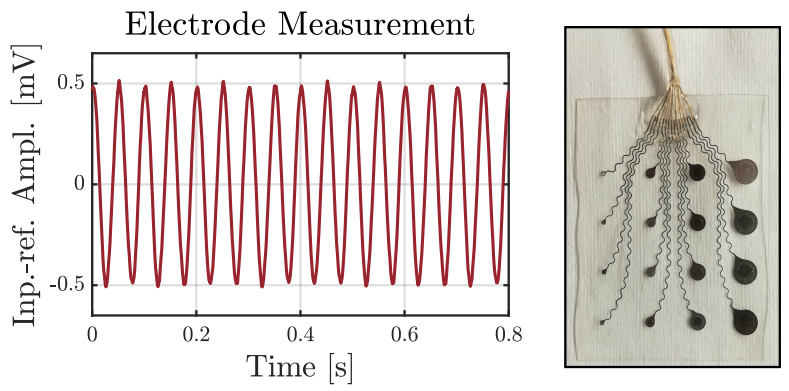

Fig. 15: Input-referred electrode measurement of a $20 \mathrm{~Hz}$, $1 \mathrm{mV}_{\mathrm{pp}}$ input sinewave, and 16-channel PtIr-electrode array with diameters of 0.5 to $2.7 \mathrm{~mm}$. The plotted recording was obtained with $1.7 \mathrm{~mm}$ electrode in PBS.

buffered saline (PBS) solution and the signal was applied by a separate, large (for low impedance) Pt-electrode. The recorded waveform was first-order low-pass filtered with $f_{\mathrm{c}}=$ $250 \mathrm{~Hz}$ post-recording, in order to restrict the in-band to the desired LFP domain. The plotted signal was divided by the measured mid-band gain of $A=39.3 \mathrm{~dB}$ for an input-referred perspective. Measurements were successfully conducted for all electrode diameters; the plotted waveform was extracted using a $1.7 \mathrm{~mm}$ electrode.

\section{StATE-OF-THE-ART COMPARISON}

Table I summarizes the measured performance of the presented neural recorder including the novel input impedance boosting scheme and allows a comparison against the state of the art. The input impedance boosting factor of 30-fold is well comparable with what is achieved using similar prechargebased methods [14], [15]. Further improvements are possible with the $C_{\text {aux }}$ precharge assist, which is fully compatible to the herein presented architecture such that the same benefits to $R_{\text {in,boost }}$ are exploitable at increased area cost.

The presented recorder has comparably larger power consumption per channel, which is due to the fact that full-band recording, low noise and low area consumption were targeted simultaneously. In [14] and [15], power is optimized at the cost of increased thermal noise, resulting in larger inputreferred noise especially in the thermal noise dominated AP range. The design reported in [25] combines very good area and power consumption with low noise, but does not feature full-band recording. The direct conversion architectures in [21], [24] allow to have infinite dc input impedance without a booster, but the area consumption is larger (however with the ADC already included) despite using a smaller CMOS node in [21] and furthermore the input referred noise is significantly larger.

The measured CMRR and PSRR compare well against the state of the art in the in-band region where the most dominant disturbers, i.e. power supply tones at multiples of $50 / 60 \mathrm{~Hz}$, are expected. Slight degradations at the band-edges are less critical, but are nevertheless included in the table. 


\begin{tabular}{|c|c|c|c|c|c|c|c|c|}
\hline & $\begin{array}{c}\text { TBioCAS'18 } \\
{[3]}\end{array}$ & $\begin{array}{c}\text { ISSCC'16 } \\
{[14]}\end{array}$ & $\begin{array}{c}\text { JSSC'17 } \\
\text { [15] }\end{array}$ & $\begin{array}{l}\text { CICC'18 } \\
{[12]}\end{array}$ & $\begin{array}{c}\text { JSSC'19 } \\
\text { [24] }\end{array}$ & $\begin{array}{l}\text { JSSC'20 } \\
{[21]}\end{array}$ & $\begin{array}{l}\text { JSSC'20 } \\
{[25]}\end{array}$ & $\begin{array}{l}\text { This } \\
\text { work }\end{array}$ \\
\hline $\begin{array}{r}\text { Tech. [nm] } \\
\text { Chopped Arch. } \\
\text { Analog Supply }[\mathrm{V}] \\
\text { Area/Ch. }\left[\mathrm{mm}^{2}\right] \\
\text { Power/Ch. }[\mu \mathrm{W}] \\
\text { Bandwidth }[\mathrm{Hz}]\end{array}$ & $\begin{array}{c}180 \\
\text { Yes } \\
1.8 \\
0.22 \\
3.26 \\
0.59-117\end{array}$ & $\begin{array}{c}40 \\
\text { Yes } \\
1.2 \\
0.071 \\
2 \\
0.2-5000\end{array}$ & $\begin{array}{c}40 \\
\text { Yes } \\
1.2 \\
0.069 \\
2.8 \\
0.12-5000\end{array}$ & $\begin{array}{c}180 \\
\text { Yes } \\
1.8 \\
0.201 \\
3.24 \\
0.35-5400\end{array}$ & $\begin{array}{c}180 \\
\text { Yes }^{*} \\
1.8 \\
0.088 \\
23^{* *} \\
5000\end{array}$ & $\begin{array}{c}110 \\
\text { No } \\
1 \\
0.078 \\
6.5^{* *} \\
10000\end{array}$ & $\begin{array}{c}130 \\
\mathrm{No}^{\ddagger \neq} \\
1.2 \\
0.023 \\
1.7 \\
<1-1000\end{array}$ & $\begin{array}{c}180 \\
\text { Yes } \\
1.8 \\
0.036 \\
13.7 \\
<0.1-5000\end{array}$ \\
\hline $\begin{array}{l}\text { Inp. Imp. (dc) }[\mathrm{M} \Omega] \\
\text { Imp. Boost Arch. } \\
\text { Imp. Boost }[\mathrm{x}-\text { fold }]\end{array}$ & - & $\begin{array}{l}300 \\
\text { aux. path } \\
15\end{array}$ & $\begin{array}{c}1600 \\
\text { aux. path } \\
76\left(29^{\ddagger}\right)\end{array}$ & $\begin{array}{l}440 \\
\text { pos. fb. } \\
88\end{array}$ & $\frac{\infty}{-}$ & - & $\begin{array}{l}1465 \\
- \\
-\end{array}$ & $\begin{array}{l}175 \\
\text { aux. path } \\
30\end{array}$ \\
\hline $\begin{array}{r}\text { LFP Inp.-ref. Noise }\left[\mu \mathrm{V}_{\mathrm{rms}}\right] \\
\text { NIB }[\mathrm{Hz}]\end{array}$ & $\begin{array}{c}2.02 \\
0.59-117\end{array}$ & $\begin{array}{c}2 \\
1-200\end{array}$ & $\begin{array}{c}1.8 \\
1-200\end{array}$ & $\begin{array}{c}0.65 \\
0.3-200\end{array}$ & $\begin{array}{c}3.5 \\
1-1250\end{array}$ & - & $\begin{array}{c}1.6 \\
1-500\end{array}$ & $\begin{array}{c}1.32 \\
1-200\end{array}$ \\
\hline $\begin{array}{r}\text { AP Inp.-ref. Noise }\left[\mu \mathrm{V}_{\mathrm{rms}}\right] \\
\text { NIB }[\mathrm{kHz}]\end{array}$ & - & $0.2^{7}-5$ & $\begin{array}{c}5.3 \\
0.2-5\end{array}$ & $\begin{array}{c}2.14 \\
0.2-5\end{array}$ & $\begin{array}{c}7.3 \\
0.001-5\end{array}$ & $\begin{array}{c}9.3^{\dagger} \\
0-10\end{array}$ & - & $\begin{array}{c}3.36 \\
0.2-10\end{array}$ \\
\hline $\begin{array}{r}\mathrm{NEF} \\
\text { THD }[\mathrm{dB}] \\
\text { CMRR }[\mathrm{dB}] \\
\text { PSRR }[\mathrm{dB}] \\
\text { Max. EDO }[\mathrm{mV}]\end{array}$ & $\begin{array}{c}3.36 \\
-41.7 \\
67.1 \\
69.6 \\
-\end{array}$ & $\begin{array}{c}4.9(\mathrm{AP}) \\
-74 \\
- \\
- \\
\pm 50\end{array}$ & $\begin{array}{c}4.4(\mathrm{AP}) \\
-76 \\
\geq 78 \\
\geq 76 \\
\pm 50\end{array}$ & $\begin{array}{c}1.56(\mathrm{AP}) \\
-61 \\
- \\
- \\
\pm 50\end{array}$ & $\begin{array}{c}14.2^{* *} \\
-77 \\
90 \\
92 \\
\pm 100\end{array}$ & $\begin{array}{l}9.3^{* *} \\
<-90 \\
76 \\
- \\
\pm 150^{\dagger \dagger}\end{array}$ & $\begin{array}{c}2.86\left(9.8^{* *}\right) \\
-70 \\
\geq 70 \\
- \\
\pm 500\end{array}$ & $\begin{array}{c}4.77(\mathrm{AP}) \\
-65 \\
77(84 @ 50 \mathrm{~Hz}) \\
60(83 @ 50 \mathrm{~Hz}) \\
\pm 100^{\dagger \dagger}\end{array}$ \\
\hline
\end{tabular}

TABLE I: State of the Art comparison table

For better comparability, the noise efficiency factor (NEF, [26]) is a popular metric, as it contains the mutually contradicting design parameters (thermal) noise, power and bandwidth. However, the area consumption is not included thus limiting the expressiveness of NEF alone, given that trade-offs between noise and area are possible especially in the $1 / \mathrm{f}$ noise dominated LFP domain. In the table, the bandwidth (BW) of each system is included alongside the noise integration bandwidth (NIB), where NIB $>$ BW should be fulfilled to obtain realistic values for the input referred noise and in extension the NEF. Keeping this in mind, and comparing the NEF of the listed recorders reveals that the presented design is well comparable to the state of the art in the AP range, indicating that the large power consumption produces similar benefit in the thermal noise performance. As NEF is defined for thermal noise, and noise performance is limited by $1 / f$ noise in the LFP domain, only the AP NEF is presented if specified in the respective publication. While this design's total integrated LFP noise compares very well against prior art, [12] achieves superior noise performance in the LFP range, however at the cost of a significantly larger (6fold) area consumption, where more than half of the total area is required for the DSL, which is typcially a main residual $1 / \mathrm{f}$ noise contributor.

\section{CONCLUSION}

This article revised and extended the analysis of auxiliary path input impedance boosting schemes for chopped neural recorders and derived a simple model taking into account the most pronounced buffer non-idealities. This models predicts the presence of constant current that is independent of input signals and is instead proportional to time-averaged buffer offset. Measurement results confirm that this current can be mitigated by using the presented buffer architecture which employs reconfiguration to self-compensate its mismatchbased offset. This buffer architecture is advantageous in bio-sensing, as electrode aging due to continuous charge transfer is significantly reduced; furthermore, the periodic reconfiguration mechanism can be used to minimize buffer offset in various applications, as long as time-averaging of the output is acceptable. This is typically given when the buffer output is integrated on a capacitor, as is the case in many sensor applications.

\section{REFERENCES}

[1] M. Haas, U. Bihr, J. Anders, and M. Ortmanns, "A bidirectional neural interface IC with high voltage compliance and spectral separation," in 2016 IEEE International Symposium on Circuits and Systems (ISCAS), May 2016, pp. 2743-2746.

[2] R. Shulyzki, K. Abdelhalim, A. Bagheri, M. T. Salam, C. M. Florez, J. L. P. Velazquez, P. L. Carlen, and R. Genov, "320-Channel Active Probe for High-Resolution Neuromonitoring and Responsive Neurostimulation," IEEE Transactions on Biomedical Circuits and Systems, vol. 9, no. 1, pp. 34-49, 2015.

[3] C. Wu, C. Cheng, and Z. Chen, "A 16-Channel CMOS ChopperStabilized Analog Front-End ECoG Acquisition Circuit for a ClosedLoop Epileptic Seizure Control System," IEEE Transactions on Biomedical Circuits and Systems, vol. 12, no. 3, pp. 543-553, 2018.

[4] H. Kassiri, S. Tonekaboni, M. T. Salam, N. Soltani, K. Abdelhalim, J. L. P. Velazquez, and R. Genov, "Closed-Loop Neurostimulators: A Survey and A Seizure-Predicting Design Example for Intractable Epilepsy Treatment," IEEE Transactions on Biomedical Circuits and Systems, vol. 11, no. 5, pp. 1026-1040, 2017.

[5] A. Wagle Shukla and M. S. Okun, "State of the Art for Deep Brain Stimulation Therapy in Movement Disorders: A Clinical and Technological Perspective," IEEE Reviews in Biomedical Engineering, vol. 9, pp. 219-233, 2016.

[6] A. Mohammed, M. Zamani, R. Bayford, and A. Demosthenous, "Toward On-Demand Deep Brain Stimulation Using Online Parkinson's Disease Prediction Driven by Dynamic Detection," IEEE Transactions 
on Neural Systems and Rehabilitation Engineering, vol. 25, no. 12, pp. 2441-2452, 2017.

[7] X. Liu, M. Zhang, H. Hao, A. G. Richardson, T. H. Lucas, and J. Van der Spiegel, "Wireless Sensor Brain Machine Interfaces for Closed-loop Neuroscience Studies," in 2019 IEEE 13th International Conference on ASIC (ASICON), 2019, pp. 1-4.

[8] J. L. Collinger, B. Wodlinger, J. E. Downey, W. Wang, and A. B. Schwartz, "High-performance neuroprosthetic control by an individual with tetraplegia," The Lancet, vol. 381, pp. 557-564, 2013.

[9] I. Williams, E. Brunton, A. Rapeaux, Y. Liu, S. Luan, K. Nazarpour, and T. G. Constandinou, "SenseBack - An Implantable System for Bidirectional Neural Interfacing," IEEE Transactions on Biomedical Circuits and Systems, vol. 14, no. 5, pp. 1079-1087, 2020.

[10] B. C. Johnson, S. Gambini, I. Izyumin, A. Moin, A. Zhou, G. Alexandrov, S. R. Santacruz, J. M. Rabaey, J. M. Carmena, and R. Muller,

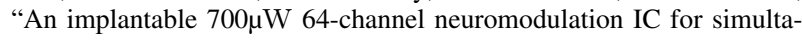
neous recording and stimulation with rapid artifact recovery," in 2017 Symposium on VLSI Circuits, 2017, pp. C48-C49.

[11] M. Haas, P. Vogelmann, and M. Ortmanns, "A Neuromodulator Frontend With Reconfigurable Class-B Current and Voltage Controlled Stimulator," IEEE Solid-State Circuits Letters, vol. 1, no. 3, pp. 54-57, 2018.

[12] D. Luo, M. Zhang, and Z. Wang, "Design of a $3.24 \mu \mathrm{W}, 39 \mathrm{nV} / \mathrm{rtHz}$ chopper amplifier with $5.5 \mathrm{~Hz}$ noise corner frequency for invasive neural signal acquisition," in 2018 IEEE Custom Integrated Circuits Conference (CICC), 2018, pp. 1-4.

[13] J. Liu and R. M. Walker, "A Compact, Low-Noise, Chopped FrontEnd for Peripheral Nerve Recording in $180 \mathrm{~nm}$ CMOS," in 2018 IEEE Biomedical Circuits and Systems Conference (BioCAS), 2018, pp. 1-4.

[14] H. Chandrakumar and D. Marković, "5.5 A $2 \mu \mathrm{W} 40 \mathrm{mVpp}$ linearinput-range chopper- stabilized bio-signal amplifier with boosted input impedance of $300 \mathrm{M} \Omega$ and electrode-offset filtering," in 2016 IEEE International Solid-State Circuits Conference (ISSCC), Jan 2016, pp. 96-97.

[15] — “ "An 80-mVpp Linear-Input Range, 1.6-G $\Omega$ Input Impedance, Low-Power Chopper Amplifier for Closed-Loop Neural Recording That Is Tolerant to 650-mVpp Common-Mode Interference," IEEE Journal of Solid-State Circuits, vol. 52, no. 11, pp. 2811-2828, Nov 2017.

[16] R. Muller, H. Le, W. Li, P. Ledochowitsch, S. Gambini, T. Bjorninen, A. Koralek, J. M. Carmena, M. M. Maharbiz, E. Alon, and J. M. Rabaey, "A Minimally Invasive 64-Channel Wireless $\mu$ ECoG Implant," IEEE Journal of Solid-State Circuits, vol. 50, no. 1, pp. 344-359, 2015.

[17] Q. Fan, F. Sebastianen, H. Huijsing, and K. Makinwa, "A $2.1 \mu \mathrm{W}$ area-efficient capacitively-coupled chopper instrumentation amplifier for ECG applications in $65 \mathrm{~nm}$ CMOS," in 2010 IEEE Asian SolidState Circuits Conference, Nov 2010, pp. 1-4.

[18] S. Reich, M. Sporer, and M. Ortmanns, "A Self-Compensated, LowOffset Voltage Buffer for Input Impedance Boosting in Chopped Neural Front-Ends," in 2020 IEEE International Symposium on Circuits and Systems (ISCAS), 2020, pp. 1-5.

[19] A. Bagheri, M. T. Salam, J. L. Perez Velazquez, and R. Genov, "Low-Frequency Noise and Offset Rejection in DC-Coupled Neural Amplifiers: A Review and Digitally-Assisted Design Tutorial," IEEE Transactions on Biomedical Circuits and Systems, vol. 11, no. 1, pp. 161-176, Feb 2017.

[20] C. Kim, S. Joshi, H. Courellis, J. Wang, C. Miller, and G. Cauwenberghs, "Sub- $\mu$ Vrms-Noise Sub- $\mu$ W/Channel ADC-Direct Neural Recording With 200-mV/ms Transient Recovery Through Predictive Digital Autoranging," IEEE Journal of Solid-State Circuits, vol. 53, no. 11, pp. 3101-3110, 2018.

[21] C. Lee, T. Jeon, M. Jang, S. Park, J. Kim, J. Lim, J. H. Ahn, Y. Huh, and Y. Chae, "A 6.5- $\mu$ W 10-kHz BW 80.4-dB SNDR Gm-CBased CT $\Delta \Sigma$ Modulator With a Feedback-Assisted Gm Linearization for Artifact-Tolerant Neural Recording," IEEE Journal of Solid-State Circuits, vol. 55, no. 11, pp. 2889-2901, 2020.

[22] H. Chandrakumar and D. Marković, "A Simple Area-Efficient RippleRejection Technique for Chopped Biosignal Amplifiers," IEEE Transactions on Circuits and Systems II: Express Briefs, vol. 62, no. 2, pp. 189-193, 2015.

[23] J. Oreggioni, A. A. Caputi, and F. Silveira, "Current-Efficient Preamplifier Architecture for CMRR Sensitive Neural Recording Applications,"
IEEE Transactions on Biomedical Circuits and Systems, vol. 12, no. 3, pp. 689-699, 2018.

[24] A. Nikas, S. Jambunathan, L. Klein, M. Voelker, and M. Ortmanns, "A Continuous-Time Delta-Sigma Modulator Using a Modified Instrumentation Amplifier and Current Reuse DAC for Neural Recording," IEEE Journal of Solid-State Circuits, vol. 54, no. 10, pp. 2879-2891, 2019.

[25] M. Reza Pazhouhandeh, M. Chang, T. A. Valiante, and R. Genov, "Track-and-Zoom Neural Analog-to-Digital Converter With Blind Stimulation Artifact Rejection," IEEE Journal of Solid-State Circuits, vol. 55, no. 7, pp. 1984-1997, 2020.

[26] M. S. J. Steyaert and W. M. C. Sansen, "A micropower low-noise monolithic instrumentation amplifier for medical purposes," IEEE Journal of Solid-State Circuits, vol. 22, no. 6, pp. 1163-1168, 1987.

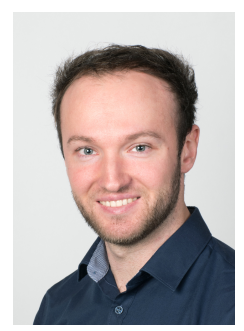

Stefan Reich (S'17) received the B.Sc. and M.Sc. in electrical engineering at the University of Ulm, Ulm, Germany, in 2015 and 2018, respectively, where he is currently pursuing the Ph.D. degree at the Institute of Microelectronics.

His research under supervision of Prof. Dr.-Ing. M. Ortmanns focuses on integrated analog and mixed-signal circuits for biomedical application and brain-machine interfaces. Starting in 2016, Mr. Reich completed an 8-month internship at the Robert Bosch Research and Technology Center in Palo Alto, CA, USA, where he worked on integrated circuits and digital synthesis.

Mr. Reich was recipient of the VDE award for his bachelor thesis in 2016 and the VDI award for his master thesis in 2019.

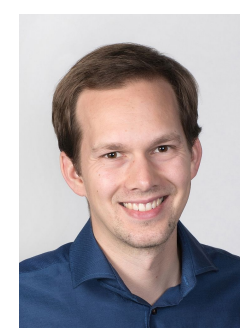

Markus Sporer (S'17) received the B.Sc. and M.Sc. degree in electrical engineering at the University of Ulm, Ulm, Germany, in 2015 and 2018, respectively, where he is currently pursuing the Ph.D. degree with the Institute of Microelectronics, under the supervision of Prof. Dr.-Ing. M. Ortmanns, with a focus on analog and mixed-signal circuits for biomedical applications.

From 2016 to 2017, he interned in the IC group at the Robert Bosch Research and Technology Center in Palo Alto, California, USA. In 2019, he joined the Institute of Microelectronics at the University of Ulm.

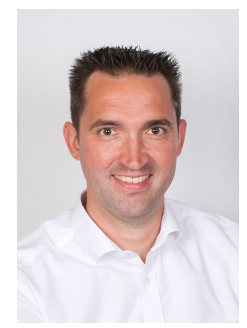

Maurits Ortmanns (M'04-SM'11) received the Dr.-Ing. degree from the University of Freiburg, Breisgau, Germany, in 2004.

From 2004 to 2005, he was with Sci-Worx $\mathrm{GmbH}$, Hannover, Germany, working in the field of mixed-signal circuits for biomedical implants. In 2006, he joined the University of Freiburg, as an Assistant Professor. Since 2008, he has been a Full Professor with the University of Ulm, Ulm, Germany, where he is currently the Head of the Institute of Microelectronics. He has authored the textbook Continuous-Time Sigma-Delta A/D Conversion, authored or coauthored several other book chapters, and over 250 IEEE journal and conference papers. He holds several patents. His current research interests include mixed-signal integrated circuit design with special emphasis on data converters and biomedical applications. 